\title{
Relations for moments of generalized record values from additive Weibull distribution and associated inference
}

\author{
R.U. Khan*, M.A. Khan, M.A.R. Khan \\ Department of Statistics and Operations Research, Aligarh Muslim University, India.
}

(Received: 29 June 2016; Accepted: 12 February 2017)

\begin{abstract}
In this note we give some simple recurrence relations satisfied by single and product moments of $k$-th upper record values from the additive Weibull distribution. These relations are deduced for moments of upper record values. Further, conditional expectation and recurrence relation for single moments are used to characterize the additive Weibull distribution and some computational works are also carried out.
\end{abstract}

Keywords Order statistics, $k$-th upper record values, additive Weibull distribution, single moments, product moments, recurrence relations and characterization

AMS 2010 subject classifications $62 \mathrm{G} 30,62 \mathrm{E} 10,60 \mathrm{E} 05$.

DOI: $10.19139 /$ soic.v5i2.237

\section{Introduction}

The statistical study of record values in a sequence of independent and identically distributed (iid) continuous random variables was first carried out by Chandler [5]. For an extensive study in this area one can refer to the works of Arnold et. al [3], Ahsanullah [1] and Ahsanullah and Nevzorov [2]. Dziubdziela and Kopociński [7] have generalized the concept of record values of Chandler [5] by random variables of a more generalized nature and we may call them as generalized record values or $k$-th record values. Setting $k=1$, we obtain ordinary record statistics.

Record values and associated statistics are of great importance in several real life problems involving weather, economic studies, sports and so on. The prediction of a future record value is an interesting problem with many real life applications. For example the predicted value of the amount of next record level of water that a dam will capture from rain and hold or discharge is helpful for future planning purposes, predicted intensity of the next strongest earthquake is essential for disaster management planning, prediction of next level of new record in athletic events is helpful for subjecting the prospective athletes to rigorous training and practice and so on.

Several applications of $k$-th record values can be found in the literature, for instance, see the examples cited in Kamps [11] or Danielak and Raqab [6] in reliability theory. Suppose that a technical system or piece of equipment is subject to shocks, e.g. peaks of voltages. If the shocks are viewed as realizations of an iid sequence, then the model of ordinary records is adequate. If it is not the records themselves, but second or third values are of special interest, then the model of $k$-th record values is adequate. When record values themselves are viewed as outliers, then the second or third largest values are of special interest. Record statistics are applied in estimating strength of materials, predicting natural disasters, sport achievements, etc. For statistical inference based on ordinary records,

\footnotetext{
${ }^{*}$ Correspondence to: Department of Statistics and Operations Research, Aligarh Muslim University, Aligarh-202 002, India. E-mails:
} aruke@rediffmail.com, khanazam2808@gmail.com

ISSN 2310-5070 (online) ISSN 2311-004X (print)

Copyright (C) 2017 International Academic Press 
serious difficulties arise if expected values of inter arrival time of records is infinite and occurrences of records are very rare in practice. This problem is avoided once we consider the model of $k$-th record statistics.

Let $\left\{X_{n}, n \geq 1\right\}$ be a sequence of iid random variables with distribution function $(d f) F(x)$ and probability density function $(p d f) f(x)$. The $j$-th order statistic of a sample $X_{1}, X_{2}, \ldots, X_{n}$ is denoted by $X_{j: n}$. For a fixed positive integer $k$, Dziubdziela and Kopociński [7] define the sequences $\left\{U_{n}^{(k)}, n \geq 1\right\}$ of $k$-th upper record times for the sequence $\left\{X_{n}, n \geq 1\right\}$ as follows:

$U_{1}^{(k)}=1$

$U_{n+1}^{(k)}=\min \left\{j>U_{n}^{(k)}: X_{j: j+k-1}>X_{U_{n}^{(k)}: U_{n}^{(k)}+k-1}\right\}$.

The sequence $\left\{Y_{n}^{(k)}, n \geq 1\right\}$, where $Y_{n}^{(k)}=X_{U_{n}^{(k)}}$ is called the sequence of generalized upper record values or $k$-th upper record values of $\left\{X_{n}, n \geq 1\right\}$. Note that for $k=1$, we have $Y_{n}^{(1)}=X_{U_{n}}, n \geq 1$, which are the record values of $\left\{X_{n}, n \geq 1\right\}$ (Ahsanullah [1]). Moreover, we see that $Y_{0}^{(k)}=0$ and $Y_{1}^{(k)}=\min \left(X_{1}, X_{2}, \ldots, X_{k}\right)=X_{1: k}$.

The $p d f$ of $Y_{n}^{(k)}$ and the joint $p d f$ of $Y_{m}^{(k)}$ and $Y_{n}^{(k)}$ are given by (Dziubdziela and Kopociński [7], Grudzień [8])

$$
f_{Y_{n}^{(k)}}(x)=\frac{k^{n}}{(n-1) !}[-\ln \bar{F}(x)]^{n-1}[\bar{F}(x)]^{k-1} f(x), \quad n \geq 1,
$$

and

$$
\begin{gathered}
f_{Y_{m}^{(k)}, Y_{n}^{(k)}}(x, y)=\frac{k^{n}}{(m-1) !(n-m-1) !}[-\ln \bar{F}(x)]^{m-1} \frac{f(x)}{\bar{F}(x)} \\
\times[\ln \bar{F}(x)-\ln \bar{F}(y)]^{n-m-1}[\bar{F}(y)]^{k-1} f(y), \quad x<y, 1 \leq m<n, n \geq 2,
\end{gathered}
$$

where

$\bar{F}(x)=1-F(x)$.

For some recent developments on generalized record values with special reference to those arising from Pareto, generalized Pareto, Weibull, exponential-Weibull and modified Weibull distributions, see Pawlas and Szynal [16, 17], Khan et. al [13] and Khan and Khan [12] respectively. In this work we mainly focus on the study of generalized record values arising from the additive Weibull distribution.

A random variable $X$ is said to have a additive Weibull distribution (Lemonte et. al [14]) if its $p d f$ is of the form

$$
f(x)=\left(\alpha \beta x^{\beta-1}+\theta \delta x^{\delta-1}\right) e^{-\left(\alpha x^{\beta}+\theta x^{\delta}\right)}, x>0, \alpha>0, \theta>0 \text { and } \delta, \beta>0
$$

and the corresponding $d f$ is

$$
F(x)=1-e^{-\left(\alpha x^{\beta}+\theta x^{\delta}\right)}, x>0, \alpha>0, \theta>0 \text { and } \delta, \beta>0 .
$$

It is easy to see that

$$
f(x)=\left(\alpha \beta x^{\beta-1}+\theta \delta x^{\delta-1}\right) \bar{F}(x) .
$$

The exponential-Weibull and Weibull distributions are the special cases for $\delta=1$ or $\beta=1$ and $\theta=0$ or $\alpha=0$, respectively. The exponential distribution arises when $\beta=1, \theta=0$ or $\alpha=0, \delta=1$. The Rayleigh and twoparameter linear failure rate distributions are obtained when $\alpha=0, \delta=2$ or $\theta=0, \beta=2$ and $\beta=2, \delta=1$ or $\beta=1, \delta=2$, respectively.

The relation in (5) will be exploited in this paper to derive some recurrence relations for the moments of $k$-th upper record values from the additive Weibull distribution and to give a characterization of this distribution.

\section{Relations for Single Moments}

Before coming to the main result we shall prove the following Lemma.

Lemma 2.1. Fix a positive integer $k \geq 1$, for $n \geq 1$ and $j=0,1, \ldots$

$$
E\left(Y_{n}^{(k)}\right)^{j}-E\left(Y_{n-1}^{(k)}\right)^{j}=\frac{j k^{n}}{(n-1) !} \int_{\alpha}^{\beta} x^{j-1}[-\ln \bar{F}(x)]^{n-1}[\bar{F}(x)]^{k} d x .
$$


Proof. From (1), we have

$$
\begin{gathered}
E\left(Y_{n}^{(k)}\right)^{j}-E\left(Y_{n-1}^{(k)}\right)^{j}=\frac{k^{n}}{(n-1) !} \int_{\alpha}^{\beta} x^{j}[-\ln \bar{F}(x)]^{n-1}[\bar{F}(x)]^{k-1} f(x) d x \\
\quad-\frac{k^{n-1}}{(n-2) !} \int_{\alpha}^{\beta} x^{j}[-\ln \bar{F}(x)]^{n-2}[\bar{F}(x)]^{k-1} f(x) d x \\
=\frac{k^{n}}{(n-1) !} \int_{\alpha}^{\beta} x^{j}[-\ln \bar{F}(x)]^{n-2}[\bar{F}(x)]^{k-1} f(x)\left\{-\ln \bar{F}(x)-\frac{n-1}{k}\right\} d x .
\end{gathered}
$$

Let

$$
h(x)=-\frac{1}{k}[-\ln \bar{F}(x)]^{n-1}[\bar{F}(x)]^{k} .
$$

Differentiating both sides of (8), we get

$$
h^{\prime}(x)=[-\ln \bar{F}(x)]^{n-2}[\bar{F}(x)]^{k-1} f(x)\left\{-\ln \bar{F}(x)-\frac{n-1}{k}\right\} d x .
$$

Thus,

$$
E\left(Y_{n}^{(k)}\right)^{j}-E\left(Y_{n-1}^{(k)}\right)^{j}=\frac{k^{n}}{(n-1) !} \int_{\alpha}^{\beta} x^{j} h^{\prime}(x) d x .
$$

Now integrating in (9) by parts and using the value of $h(x)$ from (8), we have the result given in (6).

Theorem 2.1. For distribution given in (4). Fix a positive integer $k \geq 1$, for $n \geq 1$ and $j=0,1, \ldots$

$$
E\left(Y_{n}^{(k)}\right)^{j}=\frac{\alpha \beta k}{(j+\beta)}\left\{E\left(Y_{n}^{(k)}\right)^{j+\beta}-E\left(Y_{n-1}^{(k)}\right)^{j+\beta}\right\}+\frac{\theta \delta k}{(j+\delta)}\left\{E\left(Y_{n}^{(k)}\right)^{j+\delta}-E\left(Y_{n-1}^{(k)}\right)^{j+\delta}\right\} .
$$

Proof. From (1) and (5), we have

$$
E\left(Y_{n}^{(k)}\right)^{j}=\frac{k^{n}}{(n-1) !}\left\{\alpha \beta \int_{0}^{\infty} x^{j+\beta-1}[-\ln \bar{F}(x)]^{n-1}[\bar{F}(x)]^{k} d x+\theta \delta \int_{0}^{\infty} x^{j+\delta-1}[-\ln \bar{F}(x)]^{n-1}[\bar{F}(x)]^{k} d x\right\} .
$$

Making use of Lemma 2.1, we establish the result given in (10).

\section{Remarks}

i) Setting $\delta=1$ or $\beta=1$ in (10), we get the recurrence relation for single moments of $k$-th upper record values from the exponential-Weibull lifetime distribution as obtained by Khan et. al [13].

ii) Setting $\theta=0$ or $\alpha=0$ in (10), we get the recurrence relation for single moments of $k$-th upper record values from the Weibull distribution, which verify the results obtained by Pawlas and Szynal [17].

iii) Putting $\beta=1, \theta=0$ or $\alpha=0, \delta=1$ in (10), we deduce the recurrence relation for single moments of $k$-th upper record values from the exponential distribution, established by Pawlas and Szynal [15].

iv) Putting $\alpha=0, \delta=2$ or $\theta=0, \beta=2$ in (10), we get the recurrence relation for single moments of $k$-th upper record values from the Rayleigh distribution as given by Khan et. al [13].

v) Setting $\beta=2, \delta=1$ or $\beta=1, \delta=2$ in (10), the result for single moments of $k$-th upper record values is deduced for linear failure rate distribution as established by Khan et. al [13]. 
Corollary 2.1. The recurrence relation for single moments of upper record values from the additive Weibull lifetime distribution has the form

$$
E X_{U_{n}}^{j}=\frac{\alpha \beta}{j+\beta}\left(E X_{U_{n}}^{j+\beta}-E X_{U_{n-1}}^{j+\beta}\right)+\frac{\theta \delta}{j+\delta}\left(E X_{U_{n}}^{j+\delta}-E X_{U_{n-1}}^{j+\delta}\right) .
$$

\section{Remarks}

i) If $\delta=1$ or $\beta=1$ in (11), we get the recurrence relation for single moments of record values from the exponential-Weibull lifetime distribution, obtained by Khan et. al [13].

ii) If $\theta=0$ or $\alpha=0$ in (11), the relation for single moments of record values obtained by Pawlas and Szynal [17] for Weibull distribution is deduced.

iii) If $\beta=1, \theta=0$ or $\alpha=0, \delta=1$ in (11), we get the recurrence relation for single moments of record values from exponential distribution as obtained by Balakrishnan and Ahsanullah [4] .

iv) If $\alpha=0, \delta=2$ or $\theta=0, \beta=2$ in (11), the recurrence relation for single moments of record values is deduced for Rayleigh distribution as given by Khan et. al [13].

v) If $\beta=2, \delta=1$ or $\beta=1, \delta=2$ in (11), the recurrence relation for single moments of record values is deduced for linear failure rate distribution, established by Khan et. al [13].

Numerical computations for the first four moments of upper record values from additive Weibull distribution for arbitrary chosen values of $\alpha, \beta, \theta, \delta$ and various sample size $n=1,2, \ldots, 5$ are given in Table 2.1.

Table 2.1. First four moments of upper record values

\begin{tabular}{|c|c|c|c|c|c|c|c|c|}
\hline \multirow{3}{*}{$n$} & \multicolumn{5}{|c|}{$\alpha=1, \beta=1, \theta=1$} & \multicolumn{5}{c|}{$\alpha=2, \beta=1, \theta=1$} \\
\cline { 2 - 9 } & \multicolumn{5}{|c|}{$\delta=2$} & \multicolumn{5}{c|}{$\delta=2$} \\
\cline { 2 - 9 } & $E(X)$ & $E\left(X^{2}\right)$ & $E\left(X^{3}\right)$ & $E\left(X^{4}\right)$ & $E(X)$ & $E\left(X^{2}\right)$ & $E\left(X^{3}\right)$ & $E\left(X^{4}\right)$ \\
\hline 1 & 0.54564 & 0.45435 & 0.47769 & 0.59025 & 0.37893 & 0.24212 & 0.20521 & 0.21063 \\
\hline 2 & 0.93205 & 1.06794 & 1.41557 & 2.10089 & 0.68946 & 0.62106 & 0.67101 & 0.83110 \\
\hline 3 & 1.24176 & 1.75823 & 2.76050 & 4.72074 & 0.95656 & 1.08686 & 1.40716 & 2.02388 \\
\hline 4 & 1.50624 & 2.49375 & 4.46824 & 8.56976 & 1.19363 & 1.61274 & 2.40879 & 3.91384 \\
\hline 5 & 1.74049 & 3.25950 & 6.50467 & 13.7311 & 1.40856 & 2.18286 & 3.66646 & 6.60269 \\
\hline \multirow{3}{*}{$n$} & \multicolumn{3}{|c|}{$\alpha=1, \beta=2, \theta=2$} & \multicolumn{5}{c|}{$\delta=2, \beta=2, \theta=2$} \\
\cline { 2 - 9 } & $E(X)$ & $E\left(X^{2}\right)$ & $E\left(X^{3}\right)$ & $E\left(X^{4}\right)$ & $E(X)$ & $E\left(X^{2}\right)$ & $E\left(X^{3}\right)$ & $E\left(X^{4}\right)$ \\
\hline 1 & 0.57912 & 0.39544 & 0.30227 & 0.25173 & 0.49670 & 0.29764 & 0.20235 & 0.15126 \\
\hline 2 & 0.80575 & 0.70103 & 0.64948 & 0.63459 & 0.70724 & 0.54649 & 0.45350 & 0.39951 \\
\hline 3 & 0.95933 & 0.96662 & 1.01668 & 1.11094 & 0.85302 & 0.77003 & 0.72996 & 0.72245 \\
\hline 4 & 1.07952 & 1.20789 & 1.39605 & 1.66202 & 0.96828 & 0.97703 & 1.02296 & 1.10753 \\
\hline 5 & 1.18002 & 1.43216 & 1.78391 & 2.27632 & 1.06525 & 1.17196 & 1.32804 & 1.54653 \\
\hline
\end{tabular}

\section{Relations for Product Moments}

Lemma 3.1. Fix a positive integer $k \geq 1$, for $1 \leq m \leq n-2, i, j=0,1, \ldots$ 


$$
\begin{gathered}
E\left[\left(Y_{m}^{(k)}\right)^{i}\left(Y_{n}^{(k)}\right)^{j}\right]-E\left[\left(Y_{m}^{(k)}\right)^{i}\left(Y_{n-1}^{(k)}\right)^{j}\right]=\frac{j k^{n-1}}{(m-1) !(n-m-1) !} \int_{\alpha}^{\beta} \int_{x}^{\beta} x^{i} y^{j-1} \\
\times[-\ln \bar{F}(x)]^{m-1} \frac{f(x)}{\bar{F}(x)}[\ln \bar{F}(x)-\ln \bar{F}(y)]^{n-m-1}[\bar{F}(y)]^{k} d y d x
\end{gathered}
$$

Proof. From (2), we have

$$
\begin{aligned}
& E\left[\left(Y_{m}^{(k)}\right)^{i}\left(Y_{n}^{(k)}\right)^{j}\right]-E\left[\left(Y_{m}^{(k)}\right)^{i}\left(Y_{n-1}^{(k)}\right)^{j}\right]=\frac{k^{n}}{(m-1) !(n-m-1) !} \\
& \times \int_{\alpha}^{\beta} \int_{x}^{\beta} x^{i} y^{j}[-\ln \bar{F}(x)]^{m-1} \frac{f(x)}{\bar{F}(x)}[\ln \bar{F}(x)-\ln \bar{F}(y)]^{n-m-2} \\
& \quad \times[\bar{F}(y)]^{k-1} f(y)\left\{[\ln \bar{F}(x)-\ln \bar{F}(y)]-\frac{n-m-1}{k}\right\} d y d x .
\end{aligned}
$$

Let

$$
\begin{gathered}
h(x, y)=-\frac{1}{k}[\ln \bar{F}(x)-\ln \bar{F}(y)]^{n-m-1}[\bar{F}(y)]^{k} \\
\frac{\partial}{\partial y} h(x, y)=[\ln \bar{F}(x)-\ln \bar{F}(y)]^{n-m-2}[\bar{F}(y)]^{k-1} f(y) \\
\times\left\{[\ln \bar{F}(x)-\ln \bar{F}(y)]-\frac{n-m-1}{k}\right\} .
\end{gathered}
$$

Taking into account the value of (15) in (13), we get

$$
\begin{gathered}
E\left[\left(Y_{m}^{(k)}\right)^{i}\left(Y_{n}^{(k)}\right)^{j}\right]-E\left[\left(Y_{m}^{(k)}\right)^{i}\left(Y_{n-1}^{(k)}\right)^{j}\right]=\frac{k^{n}}{(m-1) !(n-m-1) !} \\
\times \int_{\alpha}^{\beta} x^{i}[-\ln \bar{F}(x)]^{m-1} \frac{f(x)}{\bar{F}(x)}\left\{\int_{x}^{\beta} y^{j} \frac{\partial}{\partial y} h(x, y) d y\right\} d x .
\end{gathered}
$$

Now in view of (14)

$$
\int_{x}^{\beta} y^{j} \frac{\partial}{\partial y} h(x, y) d y=\frac{j}{k} \int_{x}^{\beta} y^{j-1}[\ln \bar{F}(x)-\ln \bar{F}(y)]^{n-m-1}[\bar{F}(y)]^{k} d y .
$$

After substituting (17) in (16), the required expression is obtained.

Theorem 3.1. For distribution given in (4) and $m \geq 1$ and $i, j=0,1, \ldots$

$$
\begin{gathered}
E\left[\left(Y_{m}^{(k)}\right)^{i}\left(Y_{m+1}^{(k)}\right)^{j}\right]=\frac{\alpha \beta k}{j+\beta}\left\{E\left[\left(Y_{m}^{(k)}\right)^{i}\left(Y_{m+1}^{(k)}\right)^{j+\beta}\right]-E\left(Y_{m}^{(k)}\right)^{i+j+\beta}\right\} \\
+\frac{\theta \delta k}{j+\delta}\left\{E\left[\left(Y_{m}^{(k)}\right)^{i}\left(Y_{m+1}^{(k)}\right)^{j+\delta}\right]-E\left(Y_{m}^{(k)}\right)^{i+j+\delta}\right\}
\end{gathered}
$$

and for $1 \leq m \leq n-2, i, j=0,1, \ldots$

$$
\begin{gathered}
E\left[\left(Y_{m}^{(k)}\right)^{i}\left(Y_{n}^{(k)}\right)^{j}\right]=\frac{\alpha \beta k}{j+\beta}\left\{E\left[\left(Y_{m}^{(k)}\right)^{i}\left(Y_{n}^{(k)}\right)^{j+\beta}\right]-E\left[\left(Y_{m}^{(k)}\right)^{i}\left(Y_{n-1}^{(k)}\right)^{j+\beta}\right]\right\} \\
+\frac{\theta \delta k}{j+\delta}\left\{E\left[\left(Y_{m}^{(k)}\right)^{i}\left(Y_{n}^{(k)}\right)^{j+\delta}\right]-E\left[\left(Y_{m}^{(k)}\right)^{i}\left(Y_{n-1}^{(k)}\right)^{j+\delta}\right]\right\}
\end{gathered}
$$


Proof. From (2) and (5), we have

$$
\begin{gathered}
E\left[\left(Y_{m}^{(k)}\right)^{i}\left(Y_{n}^{(k)}\right)^{j}\right]=\frac{\alpha \beta k^{n}}{(m-1) !(n-m-1) !} \int_{0}^{\infty} \int_{x}^{\infty} x^{i} y^{j+\beta-1}[-\ln \bar{F}(x)]^{m-1} \frac{f(x)}{\bar{F}(x)} \\
\times[\ln \bar{F}(x)-\ln \bar{F}(y)]^{n-m-1}[\bar{F}(y)]^{k} d y d x+\frac{\theta \delta k^{n}}{(m-1) !(n-m-1) !} \int_{0}^{\infty} \int_{x}^{\infty} x^{i} y^{j+\delta-1} \\
\times[-\ln \bar{F}(x)]^{m-1} \frac{f(x)}{\bar{F}(x)}[\ln \bar{F}(x)-\ln \bar{F}(y)]^{n-m-1}[\bar{F}(y)]^{k} d y d x .
\end{gathered}
$$

Making use of Lemma 3.1, we derive the relation given in (19). Proceeding in a similar manner for the case $n=m+1$, the recurrence relation given in (18) can easily be established.

One can also note that Theorem 2.1 can be deduced from Theorem 3.1 by putting $i=0$.

\section{Remarks}

i) Setting $\delta=1$ or $\beta=1$ in (19), we get the recurrence relation for product moments of $k$-th upper record values from the exponential-Weibull lifetime distribution as obtained by Khan et. al [13].

ii) Setting $\theta=0$ or $\alpha=0$ in (19), we get the recurrence relation for single moments of $k$-th upper record values obtained by Pawlas and Szynal [17] for Weibull distribution is deduced.

iii) Putting $\beta=1, \theta=0$ or $\alpha=0, \delta=1$ in (19), result for product moments of $k$-th upper record values is deduced for the exponential distribution as established by Pawlas and Szynal [15].

iv) Putting $\alpha=0, \delta=2$ or $\theta=0, \beta=2$ in (19), the result for product moments of $k$-th upper record values is deduced for the Rayleigh distribution as given by Khan et. al [13].

v) Setting $\beta=2, \delta=1$ or $\beta=1, \delta=2$ in (19), the result for product moments of $k$-th upper record values is deduced for linear failure rate distribution as established by Khan et. al [13].

Corollary 3.1. The recurrence relation for product moments of upper record values from the additive Weibull distribution has the form

$$
E\left(X_{U_{m}}^{i} X_{U_{n}}^{j}\right)=\frac{\alpha \beta}{j+\beta}\left\{E\left(X_{U_{m}}^{i} X_{U_{n}}^{j+\beta}\right)-E\left(X_{U_{m}}^{i} X_{U_{n-1}}^{j+\beta}\right)\right\}+\frac{\theta \delta}{j+\delta}\left\{E\left(X_{U_{m}}^{i} X_{U_{n}}^{j+\delta}\right)-E\left(X_{U_{m}}^{i} X_{U_{n-1}}^{j+\delta}\right)\right\}
$$

\section{Remarks}

i) If $\delta=1$ or $\beta=1$ in (20), we obtain the recurrence relation for product moments of record values from the exponential-Weibull lifetime distribution as established by Khan et. al [13].

ii) If $\theta=0$ or $\alpha=0$ in (20), the result for product moments of record values obtained by Pawlas and Szynal [17] for Weibull distribution is deduced.

iii) If $\beta=1, \theta=0$ or $\alpha=0, \delta=1$ in (20), the result for product moments of record values obtained by Balakrishnan and Ahsanullah [4] for the exponential distribution is deduced.

iv) If $\alpha=0, \delta=2$ or $\theta=0, \beta=2$ in (20),the recurrence relation for product moments of record values is deduced for Rayleigh distribution as given by Khan et. al [13]. 
v) If $\beta=2, \delta=1$ or $\beta=1, \delta=2$ in (20), the result for product moments of record values is deduced for linear failure rate distribution as established by Khan et. al [13].

\section{Characterizations}

Theorem 4.1. Fix a positive integer $k \geq 1$ and let $j$ be a non-negative integer. A necessary and sufficient condition for a random variable $X$ to be distributed with $p d f$ given by (3) is that

$$
E\left(Y_{n}^{(k)}\right)^{j}=\frac{\alpha \beta k}{j+\beta}\left\{E\left(Y_{n}^{(k)}\right)^{j+\beta}-E\left(Y_{n-1}^{(k)}\right)^{j+\beta}\right\}+\frac{\theta \delta k}{j+\delta}\left\{E\left(Y_{n}^{(k)}\right)^{j+\delta}-E\left(Y_{n-1}^{(k)}\right)^{j+\delta}\right\} .
$$

for $n=1,2, \ldots$

Proof. The necessary part follows immediately from (10). On the other hand if the recurrence relation (21) is satisfied, then on using (1), we have

$$
\begin{gathered}
\frac{k^{n}}{(n-1) !} \int_{0}^{\infty} x^{j}[-\ln \bar{F}(x)]^{n-1}[\bar{F}(x)]^{k-1} f(x) d x \\
=\frac{\alpha \beta k^{n+1}}{(n-1) !(j+\beta)} \int_{0}^{\infty} x^{j+\beta}[-\ln \bar{F}(x)]^{n-1}[\bar{F}(x)]^{k-1} f(x) d x \\
-\frac{\alpha \beta k^{n}}{(n-2) !(j+\beta)} \int_{0}^{\infty} x^{j+\beta}[-\ln \bar{F}(x)]^{n-2}[\bar{F}(x)]^{k-1} f(x) d x \\
+\frac{\theta \delta k^{n+1}}{(n-1) !(j+\delta)} \int_{0}^{\infty} x^{j+\delta}[-\ln \bar{F}(x)]^{n-1}[\bar{F}(x)]^{k-1} f(x) d x \\
-\frac{\theta \delta k^{n}}{(n-2) !(j+\delta)} \int_{0}^{\infty} x^{j+\delta}[-\ln \bar{F}(x)]^{n-2}[\bar{F}(x)]^{k-1} f(x) d x .
\end{gathered}
$$

Integrating the first and third integral on right side of (22) by parts and simplifying the resulting expression, we find that

$$
\int_{0}^{\infty} x^{j}[-\ln \bar{F}(x)]^{n-1}[\bar{F}(x)]^{k-1}\left\{f(x)-\left(\alpha \beta x^{\beta-1}+\theta \delta x^{\delta-1}\right) \bar{F}(x)\right\} d x=0 .
$$

Now applying a generalization of the Müntz-Szász Theorem (see for example Hwang and Lin [10]) to (23), we obtain

$$
f(x)=\left(\alpha \beta x^{\beta-1}+\theta \delta x^{\delta-1}\right) \bar{F}(x),
$$

which proves that $f(x)$ has the form as in (5).

Remark 4.1. Theorem 4.1 can be used to characterize the exponential-Weibull lifetime and Weibull distributions by setting $\delta=1$ or $\beta=1$ and $\theta=0$ or $\alpha=0$, respectively. The exponential distribution when $\beta=1, \theta=0$ or $\alpha=0, \delta=1$. The Rayleigh and two-parameter linear failure rate distributions when $\alpha=0$ , $\delta=2$ or $\theta=0, \beta=2$ and $\beta=2, \delta=1$ or $\beta=1, \delta=2$, respectively.

Corollary 4.1. Under the assumptions of Theorem 4.1 with $j=0$ the following equations

$$
E\left(Y_{n}^{(k)}\right)^{\delta}=E\left(Y_{n-1}^{(k)}\right)^{\delta}-\frac{\alpha}{\theta}\left\{E\left(Y_{n}^{(k)}\right)^{\beta}-E\left(Y_{n-1}^{(k)}\right)^{\beta}\right\}+\frac{1}{\theta k}, \quad n=1,2, \ldots
$$

characterize the additive Weibull distribution.

Remark 4.2. If $k=1$ we obtain the following characterization of the additive Weibull distribution

$$
E X_{U_{n}}^{\delta}=E X_{U_{n-1}}^{\delta}-\frac{\alpha}{\theta}\left(E X_{U_{n}}^{\beta}-E X_{U_{n-1}}^{\beta}\right)+\frac{1}{\theta}, \quad n=1,2, \ldots .
$$


Now we shall show how the Theorem 4.1 can be used in a characterization of the additive-Weibull distribution in terms of moments of minimal order statistics. Putting $n=1$ in (21), we get

$$
E X_{1: k}^{j}=\frac{\alpha \beta k}{j+\beta} E X_{1: k}^{j+\beta}+\frac{\theta \delta k}{j+\delta} E X_{1: k}^{j+\delta},
$$

for any fixed integer $k \geq 1$. This result leads to the following theorem.

Theoram 4.2. Let $j$ be a non-negative integer. A necessary and sufficient condition for a random variable $X$ to be distributed with $p d f$ given by $(3)$ is that

$$
E X_{1: k}^{j}=\frac{\alpha \beta k}{j+\beta} E X_{1: k}^{j+\beta}+\frac{\theta \delta k}{j+\delta} E X_{1: k}^{j+\delta}
$$

for $k=1,2, \ldots$.

Proof. The necessary part follows immediately from (10).On the other hand if the recurrence relation (24) is satisfied, then

$$
\int_{0}^{\infty} x^{j}[\bar{F}(x)]^{k-1} f(x) d x=\frac{\alpha \beta k}{j+\beta} \int_{0}^{\infty} x^{j+\beta}[\bar{F}(x)]^{k-1} f(x) d x+\frac{\theta \delta k}{j+\delta} \int_{0}^{\infty} x^{j+\delta}[\bar{F}(x)]^{k-1} f(x) d x
$$

Integrating the integrals on the right-hand side in (25) by parts, we get

$$
\int_{0}^{\infty} x^{j}[\bar{F}(x)]^{k-1} f(x) d x=\alpha \beta \int_{0}^{\infty} x^{j+\beta-1}[\bar{F}(x)]^{k} d x+\theta \delta \int_{0}^{\infty} x^{j+\delta-1}[\bar{F}(x)]^{k} d x
$$

which further reduces to

$$
\int_{0}^{\infty} x^{j}[\bar{F}(x)]^{k-1}\left\{f(x)-\left(\alpha \beta x^{\beta-1}+\theta \delta x^{\delta-1}\right) \bar{F}(x)\right\} d x=0, \quad k=1,2, \ldots .
$$

Now applying a generalization of the Müntz-Szász Theorem, (see for example Hwang and Lin [10]) to (26), we obtain

$$
f(x)=\left(\alpha \beta x^{\beta-1}+\theta \delta x^{\delta-1}\right) \bar{F}(x)
$$

which proves that

$$
F(x)=1-e^{-\left(\alpha x^{\beta}+\theta x^{\delta}\right)}, \quad x>0, \alpha>0, \theta>0 \text { and } \delta, \beta>0 .
$$

Theorem 4.3. Let $X$ be a non-negative random variable having an absolutely continuous $d f F(x)$ with $F(0)=0$ and $0 \leq F(x) \leq 1$ for all $x>0$, then

$$
E\left[\xi\left(Y_{n}^{(k)}\right) \mid\left(Y_{l}^{(k)}\right)=x\right]=e^{-\left(\alpha x^{\beta}+\theta x^{\delta}\right)}\left(\frac{k}{k+1}\right)^{n-l}, \quad l=m, m+1
$$

if and only if

$$
F(x)=1-e^{-\left(\alpha x^{\beta}+\theta x^{\delta}\right)},
$$

$$
\xi(y)=e^{-\left(\alpha y^{\beta}+\theta y^{\delta}\right)},
$$

Proof. From (2) and (1), we have

$$
E\left[\xi\left(Y_{n}^{(k)}\right) \mid\left(Y_{m}^{(k)}\right)=x\right]=\frac{k^{n-m}}{(n-m-1) !} \int_{x}^{\infty} e^{-\left(\alpha y^{\beta}+\theta y^{\delta}\right)}[\ln \bar{F}(x)-\ln \bar{F}(y)]^{n-m-1}\left(\frac{\bar{F}(y)}{\bar{F}(x)}\right)^{k-1} \frac{f(y)}{\bar{F}(x)} d y .
$$

By setting $u=\frac{\bar{F}(y)}{\bar{F}(x)}=\frac{e^{-\left(\alpha y^{\beta}+\theta y^{\delta}\right)}}{e^{-\left(\alpha x^{\beta}+\theta x^{\delta}\right)}}$ from (4) in (28), we have

$$
E\left[\xi\left(Y_{n}^{(k)}\right) \mid\left(Y_{m}^{(k)}\right)=x\right]=\frac{k^{n-m}}{(n-m-1) !} e^{-\left(\alpha x^{\beta}+\theta x^{\delta}\right)} \int_{0}^{1} u^{k}(-\ln u)^{n-m-1} d u .
$$


We have Gradshteyn and Ryzhik ([6], p-551)

$$
\int_{0}^{1}(-\ln x)^{\mu-1} x^{\nu-1} d x=\frac{\Gamma \mu}{\nu^{\mu}}, \quad \mu>0, \quad \nu>0 .
$$

On using (30) in (29), we have the result given in (27).

To prove sufficient part, we have

$$
\frac{k^{n-m}}{(n-m-1) !} \int_{x}^{\infty} e^{-\left(\alpha y^{\beta}+\theta y^{\delta}\right)}[\ln \bar{F}(x)-\ln \bar{F}(y)]^{n-m-1}[\bar{F}(y)]^{k-1} f(y) d y=[\bar{F}(x)]^{k} g_{n \mid m}(x),
$$

where

$$
g_{n \mid m}(x)=e^{-\left(\alpha x^{\beta}+\theta x^{\delta}\right)}\left(\frac{k}{k+1}\right)^{n-m} .
$$

Differentiating (31) both the sides with respect to $x$, we get

$$
\begin{aligned}
& -\frac{k^{n-m} f(x)}{\bar{F}(x)(n-m-2) !} \int_{x}^{\infty} e^{-\left(\alpha y^{\beta}+\theta y^{\delta}\right)}[\ln \bar{F}(x)-\ln \bar{F}(y)]^{n-m-2} \\
& \times[\bar{F}(y)]^{k-1} f(y) d y=g_{n \mid m}^{\prime}(x)[\bar{F}(x)]^{k}-k g_{n \mid m}(x)[\bar{F}(x)]^{k-1} f(x)
\end{aligned}
$$

or

$$
-k g_{n \mid m+1}(x)[\bar{F}(x)]^{k-1} f(x)=g_{n \mid m}^{\prime}(x)[\bar{F}(x)]^{k}-k g_{n \mid m}(x)[\bar{F}(x)]^{k-1} f(x) .
$$

Therefore,

$$
\frac{f(x)}{\bar{F}(x)}=-\frac{g_{n \mid m}^{\prime}(x)}{k\left[g_{n \mid m+1}(x)-g_{n \mid m}(x)\right]}=\left(\alpha \beta x^{\beta-1}+\theta \delta x^{\delta-1}\right)
$$

where

$$
\begin{gathered}
g_{n \mid m}^{\prime}(x)=-\left(\alpha \beta x^{\beta-1}+\theta \delta x^{\delta-1}\right) e^{-\left(\alpha x^{\beta}+\theta x^{\delta}\right)}\left(\frac{k}{k+1}\right)^{n-m}, \\
g_{n \mid m+1}(x)-g_{n \mid m}(x)=\frac{1}{k} e^{-\left(\alpha x^{\beta}+\theta x^{\delta}\right)}\left(\frac{k}{k+1}\right)^{n-m},
\end{gathered}
$$

which proves that

$$
F(x)=1-e^{-\left(\alpha x^{\beta}+\theta x^{\delta}\right)}, \quad x>0, \quad \alpha>0, \quad \theta>0 \text { and } \delta, \beta>0 .
$$

\section{Acknowledgement}

The authors acknowledge their gratefulness to the Chief-Editor and learned referee for their valuable comments and suggestions.

\section{REFERENCES}

1. M. Ahsanullah, Record Statistics, Nova Science Publishers, New York, 1995.

2. M. Ahsanullah and V.B. Nevzorov, Record via Probability Theory, Atlantis Press, Paris, 2015.

3. B.C. Arnold, N. Balakrishnan and H.N. Nagaraja, Records, John Wiley, New York, 1998.

4. N. Balakrishnan and M. Ahsanullah, Relations for single and product moments of record values from exponential distribution, Journal of Applied Statistical Science, vol. 2, pp. 73-87, 1995.

5. K.N. Chandler, The distribution and frequency of record values, Journal of the Royal Statistical Society. Series B, vol. 14, pp. 220-228, 1952.

6. K. Danielak and M.Z. Raqab, Sharp bounds for expectations of $k$-th record increments, Australian and New Zealand Journal of Statistics, vol. 46, pp. 665-673, 2004.

7. W. Dziubdziela and B. Kopociński, Limiting properties of the $k$-th record value, Applicationes Mathematicae, vol. 15, pp. 187$190,1976$.

8. I.S. Gradshteyn and I.M. Ryzhik, Table of Integrals, Series and Products, Academic Press, New York, 2007. 


\section{RELATIONS FOR MOMENTS OF GENERALIZED RECORD VALUES FROM ADDITIVE WEIBULL DISTRIBUTION...}

9. Z. Grudzień, Characterization of distribution of time limits in record statistics as well as distributions and moments of linear record statistics from the samples of random numbers, Praca Doktorska, UMCS, Lublin, 1982.

10. J.S. Hwang and G.D. Lin, On a generalized moments problem II, Proceedings of the American Mathematical Society, vol. 91, pp. 577-580, 1984.

11. U. Kamps, A Concept of Generalized Order Statistics, B.G. Teubner Stuttgart, Germany, 1995.

12. M.A. Khan and R.U. Khan, $k$-th upper record values from modified Weibull distribution and characterization, International Journal of Computational and Theoretical Statistics, vol. 3, pp. 75-80, 2016.

13. R.U. Khan, A. Kulshrestha and M.A. Khan, Relations for moments of $k$-th record values from exponential-Weibull lifetime distribution and a characterization, Journal of the Egyptian Mathematical Society, vol. 23, pp. 558-562, 2015.

14. A.J. Lemonte, G.M. Cordeiro and E.M.M. Ortega, On the additive Weibull distribution, Journal of Statistical Computation and Simulation, vol. 43, pp. 2066-2080, 2014.

15. P. Pawlas and D. Szynal, Relations for single and product moments of k-th record values from exponential and Gumble distributions, Journal of Applied Statistical Science, vol. 7, pp. 53-62, 1998.

16. P. Pawlas and D. Szynal, Recurrence relations for single and product moments of $k$-th record values from Pareto, generalized Pareto and Burr distributions, Communications in Statistics - Theory and Methods, vol. 28, pp. 1699-1709, 1999.

17. P. Pawlas and D. Szynal, Recurrence relations for single and product moments of $k$-th record values from Weibull distribution and a characterization, Journal of Applied Statistical Science, vol. 10, pp. 17-26, 2000. 